\title{
Landscape and climatic variations of the Quaternary shaped multiple secondary contacts among barn owls (Tyto alba) of the Western Palearctic
}

\author{
Tristan Cumer $^{1 *}$, Ana Paula Machado ${ }^{1}$, Guillaume Dumont ${ }^{1}$, Vasileios Bontzorlos ${ }^{2,3}$, Renato \\ Ceccherelli ${ }^{4}$ Motti Charter ${ }^{5,6}$, Klaus Dichmann ${ }^{7}$, Hans-Dieter Martens ${ }^{8}$, Nicolaos Kassinis ${ }^{9}$, Rui \\ Lourenço $^{10}$, Francesca Manzia ${ }^{11}$, Laure Prévost ${ }^{12}$, Marko Rakovic ${ }^{13}$, Felipe Siverio ${ }^{14}$, \\ Alexandre Roulin ${ }^{\ddagger 1}$, Jérôme Goudet ${ }^{\ddagger 1,15}$ \\ $\ddagger$ co-senior authors \\ * corresponding author
}

Contact details for corresponding author: Department of Ecology and Evolution, Biophore 13 Building, University of Lausanne, CH -1015 Lausanne, Switzerland. Tel: +4121 6924218. Email: Tristan.cumer@unil.ch

1 Department of Ecology and Evolution, University of Lausanne, Lausanne, Switzerland 2 Green Fund, Kifisia, Athens, Greece

3 "TYTO" - Organization for the Management and Conservation of Biodiversity in Agricultural Ecosystems, Larisa, Greece 4 Centro Recupero Rapaci del Mugello, Firenze, Italy 5 Shamir Research Institute, University of Haifa, Katzrin, Israel

6 Department of Geography and Environmental Sciences, University of Haifa, Haifa, Israel 7 Hyldehegnet 27, 6400 Sønderborg, Denmark 8 Gettorfer Weg 13, 24214 Neuwittenbek, Germany 9 Game and Fauna Service, Ministry of the Interior, Nicosia, Cyprus 10 MED Mediterranean Institute for Agriculture, Environment and Development, Laboratory of Ornithology, IIFA, University of Évora, Évora, Portugal

11 Centro di Recupero per la Fauna Selvatica-LIPU, Rome, Italy

12 Association C.H.E.N.E., Centre d'Hébergement et d'Etude sur la Nature et I'Environnement, 76190 Allouville-Bellefosse, France

13 Natural History Museum of Belgrade, Belgrade, Serbia 14 Canary Islands' Ornithology and Natural History Group (GOHNIC) 38480 Buenavista del Norte, Tenerife, Canary Islands, Spain 15 Swiss Institute of Bioinformatics, Lausanne, Switzerland

Tristan Cumer - 0000-0002-0276-7462

Ana Paula Machado - 0000-0001-9203-7000

Motti Charter - 0000-0003-3861-6356

Nicolaos Kassinis - 0000-0003-2559-6683

Rui Lourenço - 0000-0001-7694-0478

Marko Rakovic - 0000-0002-3658-3362

Felipe Siverio - 0000-0002-0324-0646

Alexandre Roulin - 0000-0003-1940-6927

Jérôme Goudet - 0000-0002-5318-7601 
bioRxiv preprint doi: https://doi.org/10.1101/2021.06.09.447652; this version posted June 10, 2021. The copyright holder for this preprint

(which was not certified by peer review) is the author/funder, who has granted bioRxiv a license to display the preprint in perpetuity. It is made available under aCC-BY-NC 4.0 International license.

\section{$47 \quad$ Key words}

48

49 Demographic modeling; glacial refugium; Haplotypes; Population genomics; postglacial

50 recolonization; Whole-genome resequencing 


\section{Abstract}

52 The combined actions of climatic variations and landscape barriers shape the history of

53 natural populations. When organisms follow their shifting niches, obstacles in the landscape

54 can lead to the splitting of populations, on which evolution will then act independently. When

55 two such populations are reunited, secondary contact occurs in a broad range of admixture patterns, from narrow hybrid zones to the complete dissolution of lineages. A previous study suggested that barn owls colonized the Western Palearctic after the last glaciation in a ringlike fashion around the Mediterranean Sea, and conjectured an admixture zone in the Balkans. Here, we take advantage of whole-genome sequences of 94 individuals across the Western Palearctic to reveal the complex history of the species in the region using

61 observational and modeling approaches. Even though our results confirm that two distinct lineages colonized the region, one in Europe and one in the Levant, they suggest that it predates the last glaciation and identify a narrow secondary contact zone between the two in Anatolia. Nonetheless, we also show that barn owls re-colonized Europe after the glaciation from two distinct glacial refugia: a western one in Iberia and an eastern one in Italy. Both glacial lineages now communicate via eastern Europe, in a wide and permeable contact zone.

67 This complex history of populations enlightens the taxonomy of Tyto alba in the region, highlights the key role played by mountain ranges and large water bodies as barriers and 
Introduction

71 Species distribution patterns fluctuate in response to climatic variations, as populations

72 relocate to follow their shifting niches ${ }^{1}$. When organisms colonize new areas, obstacles in the

73 landscape may lead populations to split with varying degrees of geographic isolation.

74 Evolution, via mutation, drift, local adaptation and gene flow, will then act independently on

each of the isolated populations. If two allopatric populations are later geographically

reconnected, after a certain amount of time and divergence, it can create a secondary contact

zone. For example, when populations on both sides of an obstacle meet at the end of it in a

ring-like fashion, as described in birds ${ }^{2}$, amphibiens ${ }^{3,4}$, or plants ${ }^{5}$. Likewise, climate oscillations can lead to cyclical isolation and secondary contacts between populations as regional suitability varies ${ }^{6}$.

This complex interplay between climatic variations and landscape has been extensively studied in the Western Palearctic ${ }^{7,8}$, specifically in light of the cycles of glacial and interglacial periods that characterized the Quaternary ${ }^{9}$. During the last glaciation, colder temperatures and the expansion of the ice sheets in the north rendered large areas unsuitable for many species, which led them to follow their niches southward. Species found refuge in the Mediterranean peninsulas and in northern Africa where climatic conditions were more amenable, forming isolated populations. At the end of the last glaciation maximum (i.e. approximatively $20 \mathrm{k}$ years ago ${ }^{10}$ ), this process was reversed as the climate warmed and the melting of the continental ice caps exposed free land that could be recolonized. An extensive literature addressing the post-glacial history of European organisms describes how the complex landscape of the continent, combined with the distribution of species during the glaciation, conditioned their recolonization processes ${ }^{8,11}$. However, the low-resolution of 
genetic data used before the genomic era was often insufficient to resolve the intricate and

94 often fine-scale evolutionary processes that occurred during recolonization.

95 Rapid development of high-throughput sequencing technologies and corresponding methodological tools during the last decades has opened new avenues to study natural populations with high precision. In particular, it has allowed biologists to reconstruct the evolutionary history of species and highlight the diversity of processes acting when populations or subspecies interact in secondary contact. These processes have been found to result in a variety of situations. While the prolonged isolation of populations may lead to allopatric speciation (many examples in plants ${ }^{12,13}$, amphibian ${ }^{3}$, insects ${ }^{14}$, mammals ${ }^{15}$ and birds $\left.{ }^{16}\right)$, secondary contact tends to show a broad range of admixture patterns. When admixture occurs, it may vary from narrow hybrid zones between lineages ${ }^{17}$, to the complete dissolution of a lineage ${ }^{18}$, through a gradual level of admixture along a gradient of mixing populations ${ }^{19}$.

106 Microsatellite and mitochondrial data suggested that the barn owls (Tyto alba), a non107 migratory raptor, colonized the Western Palearctic in a ring-like fashion around the 108 Mediterranean Sea after the last glaciation ${ }^{20}$. Under this scenario, a postglacial expansion 109 from the glacial refugium in the Iberian Peninsula to northern Europe formed the western 110 branch of the ring, with the eastern branch present across the Levant and Anatolia. While 111 these observations led to conjecture of a potential admixture zone in the Balkans, the 112 available data at the time combined with the overall low genetic differentiation in this species 113 did not allow to fully resolve this question. Moreover, the peculiar genetic makeup of 114 populations in the presumed contact zone brought into question the possibility of a cryptic glacial refugium in the eastern Mediterranean peninsulas. 
116 The difficulty in resolving the post glacial expansion of barn owls is mirrored by their

117 convoluted taxonomy in the Western Palearctic. In this region, Tyto alba is classified into

118 different subspecies based on geography and plumage coloration. First, T. a. erlangeri

119 (Sclater, WL, 1921) reported in Crete, Cyprus and Middle East, may match the Levant lineage.

120 Second, T. a. alba (Scopoli, 1769) is white-colored and supposedly present in western Europe

121 and western Canary Islands, and could represent the western arm of the ring colonization. T.

122 a. guttata (Brehm, CL, 1831), the third subspecies is a dark rufous morph allegedly found in

123 Northern and Eastern Europe, in the Balkans and around the Aegean Sea. This taxonomy does

124 not match any known genetic lineage identified so far and overlaps with the area where

125 admixture between the two lineages that colonized Europe supposedly happens, making the

126 presence of a subspecies in this area puzzling in light of the history known so far.

127 Here, taking advantage of whole genome sequences of 94 individuals from all around

128 continental Europe and the Mediterranean Sea, we elucidate the demographic history of barn

129 owls in the Western Palearctic. Combining descriptive and modelling approaches based on

130 genomic and ecological data, we identify how the climatic variations and landscape of the

131 region shaped the history of this species. We also investigate how previously isolated

132 populations of barn owls interact at secondary contacts between different lineages, and

133 discuss the convoluted taxonomy with regards to their history. 
Material and methods

Samples and data preparation

Sampling, molecular and sequencing methods

138 The whole genomes of 96 individual barn owls (Tyto alba) were used in this study (table S1):

94 individuals were sampled in 11 Western Palearctic localities: Canary Islands (Tenerife

140 island - WC), Portugal (PT), France (FR), Switzerland (CH), Denmark (DK), Serbia (SB), Greece

141 (GR), Italy (IT), Aegean islands (AE), Cyprus (CY) and Israel (IS). In addition, one Eastern (Tyto

142 javanica from Singapore) and one American barn owl (Tyto furcata from California, USA) were

143 used as outgroups. Illumina whole-genome sequences of individuals from PT, FR, CH, DK and

144 the outgroups were obtained from the GenBank repository (BioProject PRJNA700797). For

145 the remaining 61 individuals, we followed a similar library preparation and sequencing

146 protocol as outlined in Machado et al. ${ }^{21}$. Briefly, genomic DNA was extracted using the

147 DNeasy Blood \& Tissue kit (Qiagen, Hilden, Germany), and individually tagged. 100bp TruSeq

148 DNA PCR-free libraries (Illumina) were prepared according to manufacturer's instructions.

149 Whole-genome resequencing was performed on multiplexed libraries with Illumina HiSeq

1502500 PE high-throughput sequencing at the Lausanne Genomic Technologies Facility (GTF,

151 University of Lausanne, Switzerland).

152

Data processing, SNP calling and technical filtering

154 The bioinformatics pipeline used to obtain analysis-ready SNPs was adapted from the

155 Genome Analysis Toolkit (GATK) Best Practices ${ }^{22}$ to a non-model organism following the 156 developers' instructions, as in Machado et al. ${ }^{21}$. Raw reads were trimmed with Trimommatic

157 v. $0.36^{23}$ and aligned to the reference barn owl genome ${ }^{21}$ with BWA-MEM v.0.7.15 ${ }^{24}$. Base 
quality score recalibration (BQSR) was performed using high-confidence calls obtained from

two independent callers - GATK's HaplotypeCaller and GenotypeGVCF v.4.1.3 and ANGSD

v.0.921 25 - as a set of "true variants" in GATK v.4.1.3.

161 Genotype calls were filtered for analyses using a hard-filtering approach as proposed for non-

162 model organisms, using GATK and VCFtools ${ }^{26}$. Calls were removed if they presented: low

163 individual quality per depth $(Q D<5)$, extreme coverage $(1100>D P>2500)$, mapping quality

164 ( $M Q<40$ and $M Q>70)$, extreme hetero or homozygosity (ExcessHet > 20 and

InbreedingCoeff $>0.9$ ) and high read strand bias (FS $>60$ and SOR $>3$ ). Then, we removed

calls for which up to $5 \%$ of genotypes had low quality $(G Q<20)$ and extreme coverage (GenDP

$<10$ and GenDP > 40). We kept only bi-allelic sites, excluded SNPs on the heterozome (Super

scaffolds 13 and $42^{21}$ ) and an exact Hardy-Weinberg test was used to remove sites that significantly departed $(p=0.05)$ from the expected equilibrium using the package

HardyWeinberg ${ }^{27,28}$ in $\mathrm{R}^{29}$, yielding a dataset of $6,448,521$ SNP (mean individual coverage: to a total of $5,151,169$ SNP for the population genomic analyses.

175 The set of 6,448,521 variants was phased in two steps. First, individual variants were phased using a read-based approach in which reads covering multiple heterozygous sites were used

177 to resolve local haplotypes. To do so, WhatsHap $v 1.0^{30}$ was run independently for each 178 individual with default parameters. Secondly, variants were statistically phased with Shape-It v4.1.2 $2^{31}$. This algorithm integrates local individual phase and applies an approach based on coalescence and recombination to statistically phase haplotypes and impute missing data. 
conditioning neighbors in the PBWT was set to 8, and the MCMC chain was run with 10 burniterations.

To assess the quality of the phasing, we examined phase accuracy by using the switch-errorrate metric ${ }^{32}$. When comparing two phasing for an individual's variants, a switch error occurs site. Thus, for each individual, we compared the true local phasing inferred form the readbased approach (WhatsHap) and the statistical phasing of this individual's variants statistically phased by Shape-It, with read-based phase information ignored only for the individual considered (same version and parameters than in the paragraph above). The final estimation of the switch error rate was done using the switchError code to compare both phasing sets (available at https://github.com/SPG-group/switchError) (fig. S1).

\section{History of barn owls around the Mediterranean Sea}

197 In order to investigate population structure among our samples, sNMF ${ }^{33}$ was run for a clustering and admixture proportions. For this analysis, SNPs were pruned for linkage disequilibrium in PLINK v1.946 ${ }^{34}$ (parameters --indep-pairwise 5010 0.1) as recommended by the authors, yielding 594,355 SNP. Treemix ${ }^{35}$ was used to calculate a drift-based tree of our populations, using this LD-pruned dataset. To detect admixture events between 
Population expected and observed heterozygosity, population-specific private alleles, population-specific rare alleles (mac $\leq 5$ ) and population-specific total number of polymorphic sites were estimated using custom R scripts on the 5,151,169 variants dataset. To account for differences in sample sizes, which ranges from 4 to 10, population-specific statistics were calculated by randomly sampling 5 individuals from the larger populations (all except FR and SB) 10 times in a bootstrap-fashion and estimating the mean and standard deviation (SD). Individual-based relatedness $(\beta)^{36}$ and inbreeding coefficient for SNP data were calculated with the R package SNPRelate ${ }^{37}$. Overall $F_{S T}$, population pairwise $F_{S T}$ and population specific $\mathrm{F}_{\mathrm{ST}}{ }^{36}$ were computed with the hierfstat package v.0.5-9 ${ }^{38}$. Confidence intervals for population specific FST were computed by dividing the SNPs into 100 blocs, and bootstrapping 100 times 100 blocks with replacement. Finally, Principal Component Analyses (PCA) were also performed with the R package SNPRelate, first with all individuals and second only with the 66 European ones (excluding WC, CY and IS).

To measure shared ancestry in the recent past between individuals, we ran fineSTRUCTURE haplotype sharing between individuals using ChromoPainter to generate a co-ancestry matrix, which records the expected number of haplotypes chunks each individual donates to another. For this ChromoPainter step, we converted phased haps files to chromopainter phase files using the impute2chromopainter.pl script provided at the makeuniformrecfile.pl script. Using the version of ChromoPainter built into 
parameters (switch rate and mutation rate). The model was then run using the estimated model on the co-ancestry matrix for 500,000 burn-in and 500,000 sampling iterations, sampling every 10,000 iterations to determine the grouping of samples with the best posterior probability.

Modeling of history of European barn owl

Maximum-likelihood demographic inference

To describe the history of barn owls in Europe, we modeled five different demographic scenarios using fastsimcoal ${ }^{40,41}$. Given the position of Italy on the PCA (fig. 1e), its high $F_{\text {ST }}$ and lower haplotype sharing with the rest of European populations (fig. 2), we tested in particular whether it could have been a cryptic glacial refugium during the last glaciation. To focus on European populations and due to computational constraints, we simplified the dataset to model the history of four populations of eight individuals (table S1): PT as representatives of the known refugium in the Iberian Peninsula ${ }^{42}$; IT and GR representing the peninsulas of Italy and Balkans, respectively; and $\mathrm{CH}$, a product of the recolonization of northern Europe from the Iberian refugium ${ }^{42}$.

248 Autosomal SNPs were filtered to retain only neutrally evolving regions by excluding SNPs

249 found in genic regions and CPG mutations ${ }^{43}$. To achieve homogeneity among SNPs, we removed all sites with missing data and excluded positions with a coverage outside two thirds of the standard deviation of the mean. We employed a parsimony approach based on the

252 Tytonidae phylogenetic tree ${ }^{44}$ to determine the ancestral state of the SNPs using the 
genomes of the two outgroups. Sites for which it was impossible to attribute a state based on

the available outgroups were discarded. The remaining 770,718 SNPs were used to produce population pairwise site frequency spectra (SFS).

Five different scenarios were tested to model the history of barn owls in continental Europe, with a special focus on the period since the last glaciation (fig. 2, fig. S8). Three models included only one refugium in the Iberian Peninsula and various possibilities of colonization scenarios, thus excluding the persistence of barn owls in a second refugium during the glaciation. The two last models included two refugia during the last glacial maximum (LGM), a western refugium in the Iberian Peninsula and an eastern refugium, in the Italian Peninsula. The models $1 R-1,1 R-2$ and $1 R-3$ included only one refugium in the lberian Peninsula. $1 R-1$ model assumed only one colonization route around the north side of the Alps (forming the $\mathrm{CH}$ population), and from there move southeast to reach first the Balkans (GR) and then Italy (IT). The two other single-refugium models ( $1 R-2$ and $1 R-3)$ assumed two distinct colonization routes, one north of the Alps to $\mathrm{CH}$ and the other south of the Alps along the Mediterranean coast to IT. 1R-2 assumes current Greece would have been colonized by owls from the Italian Peninsula, following the route along the Mediterranean coast. In $1 R-3$, Greece would have been colonized via northern Europe, while the Mediterranean expansion would have stopped

272 in Italy.

273 The last two models included a second, eastern, refugium ( $2 R-1$ and $2 R-2)$. In these models,

274 the western lineage expansion from the Iberian population would have colonized Europe before the last glaciation, thus occupying all the Mediterranean peninsulas. During the last glaciation, two distinct populations would have survived, respectively in the Iberian (western 
refugium) and Italian (eastern refugium) Peninsulas. Both models assume that northern

Europe was recolonized from the Iberian lineage after the glaciation, but they differ in the scenario of recolonization of southeastern Europe. In $2 R-1$, Greece was recolonized from the Italian refugium while in $2 R-2$ the expansion from the Iberian Peninsula would have between populations was allowed (see fig. 2 and table S2).

Wide search ranges for initial simulation parameters were allowed for population sizes, divergence times and migration rates (table S2). Each population split was preceded by an instantaneous bottleneck, in which the founding population size was drawn from a loguniform distribution between 0.01 and 0.5 proportion of current population sizes.

Demographic simulations and parameter inference were performed under a compositemaximization (EM) cycles (-M and $-\mathrm{L})$. As we do not have an accurate mutation rate for barn BP with a 3-year generation time) and scaled all other parameters relative to it using the -0 command option (using only polymorphic sites). The best-fitting scenario out of the five tested was determined based on Akaike's information criterion ${ }^{45}$ (AIC) and confirmed through the examination of the likelihood ranges of each scenario as suggested in Kocher et 
intervals $(\mathrm{Cl})$ of the inferred parameters under the best-fitting scenario. To account for LD, a block-bootstrap approach was employed as suggested by the authors ${ }^{40,41}$ : the SNPs were divided into 100 same-size blocks, and then 100 bootstrap SFS were generated by sampling these blocks with replacement. Due to computational constraints, for bootstrapping we ran 50 independent parameter inferences per bootstrapped SFS with only 10 EM cycles each, instead of 50 cycles used for comparing scenarios above. This procedure has been defined as conservative ${ }^{47}$, and is expected to produce quite large confidence intervals. We accepted this trade-off as our main goal was to determine the best demographic topology, accepting uncertainty on specific parameter values. The highest maximum-likelihood run for each bootstrapped SFS was used to estimate $95 \% \mathrm{Cl}$ of all parameters.

Niche modeling

In order to identify the regions of high habitat suitability for barn owls at the last glacial maximum (LGM, 20'000 years BP) and to support the demographic scenarios tested in the previous section, we modelled the past spatial distribution of the species in the Western Palearctic. We built species distribution model (SDM) using Maximum Entropy Modelling

317 (MaxEnt), a presence-only based tool ${ }^{48}$. Current climatic variables for the Western Palearctic

318 (fig. S10) were extracted from the WorldClim database at 5 arc min resolution using the $\mathrm{R}$ package rbioclim ${ }^{49}$, and filtered to remove variables with a correlation of 0.8 or higher (fig. 
325

326

327

328

330

331

332

333

334

335

336

337

338

339

340

341

342 map as described for current data (fig. 2c).

combination optimized the model without over complexifying it. The best combination based on the corrected AIC (as recommended by Warren \& Seifert ${ }^{50}$ ) was achieved with a quadratic model with 1 as regularization multiplier (table S5). We ran 100 independent maxent models, omitting $25 \%$ of the data during training to test the model. To avoid geographic bias due to different sampling effort in the distribution area of the species, we randomly extracted 1000 presence points within the IUCN distribution map ${ }^{51}$ for each model run ${ }^{52}$.

Predictive performances of the models were evaluated on the basis of the area under the curve $(A \cup C)$ of the receiver operator plot of the test data. For all models with an AUC higher than 0.8 (considered a good model ${ }^{53,54}$ ), we transformed the output of Maxent into binary maps of suitability. We assigned a cell as suitable when its mean suitability value was higher than the mean value of the $10 \%$ test presence threshold. This conservative threshold allows us to omit all regions with habitat suitability lower than the suitability values of the lowest $10 \%$ of occurrence records. Finally, we averaged the values of the models for each cell, and only cells suitable in $90 \%$ of the models were represented as such in the map.

We projected the models to the climatic conditions of the mid-Holocene ( $6^{\prime} 000$ years BP) and the LGM (20'000 years BP), which we extracted from WorldClim at the same resolution as current data. When projecting to past climates, the Multivariate Environmental Similarity Surface (MESS) approach ${ }^{48}$ was used to assess whether models were projected into climatic conditions different from those found in the calibration data. As our goal was to highlight only areas of high suitability for barn owls, cells with climatic conditions outside the distribution used to build the model were assigned as unsuitable ( 0 attributed to cell with negative MESS). For each timepoint, the results of the models were merged and transformed into a binary 


\section{Barriers and corridors}

Migration surface estimation in the western Palearctic

351 The Estimated Effective Migration Surface (EEMS) v.0.0.9 software ${ }^{55}$ was used to visualize geographic regions with higher or lower than average levels of gene flow between barn owl populations of the Western Palearctic. Using the SNP dataset pruned for LD produced above, we calculated the matrix of genetic dissimilarities with the tool bed2diff. The free Google Maps api v.3 application available at http://www.birdtheme.org/useful/v3tool.html was used to draw the polygon outlining the study area in the Western Palearctic. EEMS was run with 1000 demes in five independent chains of 5 million MCMC iterations with a burn-in of 1 million iterations. Results were visually checked for MCMC chain convergence (fig. S11) and through the linear relation between the observed and fitted values for within- and betweendemes estimates using the associated R package rEEMSplots v.0.0.1 ${ }^{55}$. With the same package, we produced a map of effective migration surface by merging the five MCMC chains.

364 To investigate how population structure correlated with spatial distances between European populations and to detail the role of the Alps as a barrier to gene flow, we performed Mantel tests as implemented in the ade4 package v.1.7-15 ${ }^{56}$ for R. We compared the genetic distances (pairwise Fst between populations, see section Population Structure and Genetic Diversity for details) with different measures of geographical distance between populations: the shortest distance over land via direct flight and the distance constrained by the presence of the Alps, forcing the connection of the Italian population to the other populations via the 
$\underline{\text { Results }}$

374 History of barn owls around the Mediterranean Sea

375 Genetic diversity and population structure in the Western Palearctic

376 Despite an overall low differentiation (overall $F_{\mathrm{ST}}=0.047$, comparable with the overall

377 FST=0.045 estimated by Burri et al. ${ }^{20}$ ), the dataset revealed a structuration of the genetic

378 diversity among barn owls of the Western Palearctic. The first axis of the genomic PCA

379 (explaining 3.32\% of the total variance) contrasted individuals from the Levant populations

380 (IS and CY) to all other individuals (fig. 1d), consistent with $\mathrm{K}=2$ being the best estimate in sNMF (fig. S4, fig. S5). For K=3 (fig. 1b), the Canary population (WC) formed an independent genetic cluster, and this was confirmed by the second axis of the PCA (explaining $2.6 \%$ of the variance) opposing it to all other individuals (fig. 1d, fig. S2). This isolation of WC was also observable in table 1 , with a lot of privates and rare alleles in Tenerife island, it's higher $F_{I T}$ and the highest population specific Fst of all sampled populations. On the same PCA (fig. 1d), individuals from European populations ( $F R, C H, D K, I T, S B, G R, A E$ ) formed a third distinct cluster, matching their grouping in a single cluster at $\mathrm{K}=3$ with sNMF (fig. 1b). The Iberian individuals (PT) occupied a central position on the PCA (around 0 on both axes) and a mixed composition in sNMF (fig. 1b). This central position of the Iberian population was also visible in the pairwise $F_{\mathrm{ST}}$, where the highest value in the pairs involving PT is 0.055 (with both $\mathrm{CY}$ and WC) while all other pairwise comparisons involving populations from two of the distinct groups identified before (Levant, Canary Islands and Europe) have values equal or higher (fig. 
the Levant populations (IS and CY) in a second lineage and finally all European populations in a third lineage. In Europe, the Iberian population was basal to all other populations.

399 Focusing only on European samples, southern populations (PT, IT, GR and AE) harbored a

412 European PCA. For $k=5$, a third European component was distinguished in the Aegean

413 individuals. This component was the majoritarian in Greek samples with contributions of both

414 northern and Italian component; and Serbian samples appeared as a mix of the northern and 
419 TreeMix was from $\mathrm{CY}$, a population from the Levant lineage, to $\mathrm{AE}$, a population from the

420 European lineage (fig. 1f).

421

422

Fine Structure and Haplotype sharing

423 The clustering of individuals by FineStructure, based on shared haplotypes between

424 individuals was consistent with previous results (fig. S7). Individuals from the different

425 populations sampled were monophyletic, except for $\mathrm{CH}$ and FR individuals, mixed in the same

426 population. Consistently with this grouping, haplotypes from any given population were more

427 likely to be found in individuals from the same population, followed by its most related 428 populations (fig. 2). In the Levant lineage, IS haplotypes mostly painted IS Individuals but also

$429 \mathrm{CY}$ individuals and vice versa. Iberian haplotypes mostly painted PT individuals, but also 430 contributed greatly to the painting of all European individuals, decreasing with distance.

431 Western European haplotypes (from FR and $\mathrm{CH}$ ) mostly painted western European

432 individuals, then northern individuals (from DK) and finally eastern individuals (from SB, GR

433 and $A E)$. The reverse pattern was observed for haplotypes from eastern Europe (GR, AE), with

434 a gradient of contribution decreasing from east to west. Haplotypes from DK and SB mostly

435 painted individuals from their own population, but also in their respective neighbors in both

436 eastern and western European populations. Italian haplotypes were the most distinct

437 haplotypes among European populations, mostly painting Italian individuals, followed by

438 Greek individuals, and being painted by other populations at a lower rate than expected given

439 its geographic position. Finally, AE haplotypes also painted more often CY individuals than IS

440 individuals. This painting of levant individuals by AE haplotypes was higher than the

441 contribution from any other European individual, and both $\mathrm{CY}$ and IS haplotypes painted more

$442 \quad A E$ individuals than any other European individual. 


\section{Modeling of the history of European barn owls}

445

446

447

448

449

450

451

452

453

454

455

456

457

458

459

460

461

462

463

464

465

466

\section{Species distribution modeling}

Habitat suitability projections showed that, from a climatic point of view, there were suitable regions for barn owls all around the Mediterranean Sea during the glaciation (20'000 years BP; fig. 3c). Large areas were suitable in northern Africa and the Iberian Peninsula, but also in the two eastern Mediterranean peninsulas (current Italy and Greece). At this point, the sea levels were lower than today's and the two eastern peninsulas were more connected, allowing for a continuous region of suitable barn owl habitat. At the mid-Holocene $\left(6^{\prime} 000\right.$ years BP), major changes in sea level revealed a coastline very similar to nowadays. Our projections revealed a reduction of habitat suitability in northern Africa at this time, while the suitability of western and northern Europe increased (fig. 3c). Finally, today, nearly all continental Europe is suitable for the barn owls, with the notable exception of mountain areas (fig. 3c).

\section{Demographic inference}

AIC and raw likelihood comparisons showed that the two refugia model $2 R-1$ explains best the SFS of our dataset (table S3; Fig. 3b). In this model, an ancestral Italian lineage (IT) split from the Iberian lineage (PT) before the last glaciation, estimated at approximately 69'000 years BP (95\% Cl: 24'000-90'000 years BP; calculated with 3-year generation time). After its initial expansion, the ancestral population is estimated to have been larger in the Italian Peninsula that in Iberia (respectively $189 \mathrm{~K}$ (11K-320k) and 11k (10K-73k) haploid individuals). During the glaciation (fixed between 24 and $18 \mathrm{~K}$ years BP), both populations experienced a bottleneck, with a population size reduced to $6.8 \mathrm{k}(1.2 \mathrm{k}-141 \mathrm{k})$ individuals in the Iberian 
lineage and $62(36-116 \mathrm{k})$ in the Italian. After the glaciation, the size of both populations increased to their current size, estimated at $44 \mathrm{k}$ (20K-380k) in the Iberian Peninsula and 1.3k

469 (1k-326k) in the Italian Peninsula, both smaller but consistent with their estimated census size 470 (55k-98k 57,58 and $6 k-13 k^{59}$, respectively). The Greek population split from the Italian branch

471 around 5'700 years BP while the Swiss $(\mathrm{CH})$ population split slightly later from Iberia $\left(5^{\prime} 000\right.$

472 years BP) and maintain a high level of gene flow (estimated to 90 (46-1.4k) from CH to PT and

$4738(3-62)$ in the reverse direction). Current effective population sizes of the $\mathrm{CH}$ and GR 474 populations are estimated to $3.4 k(1 k-205 k)$ and $1.4 k(1 k-208 k)$, respectively (fig. $2 b)$, in line 475 with census results (1000-2500 60 and 3000-6000 ${ }^{61}$, respectively). Migration between these 476 populations is estimated to be highest from IT and GR to $\mathrm{CH}$ (respectively 27 (0.2-157)

477 migrants from IT and 42 (0.1-156) from GR) and lowest in the opposite direction (respectively $4781.3(0.1-96)$ migrants from $\mathrm{CH}$ to $\mathrm{GR}$ and $0.02(0.2-38)$ from $\mathrm{CH}$ to IT) (table S4). Point 479 estimates with 95\% confidence intervals for all parameters of the best model are given in 480 (table S4), as well as single point estimates for all models (table S2).

\section{Barriers and corridors}

Migration Surface Estimate in the Western Palearctic

484 Estimated Effective Migration Surface identified large water bodies, especially in the eastern Mediterranean and around Cyprus, as regions resisting to migration (fig. S12). On the mainland, barriers to gene flow matched the main formations of the Alpide belt in the region, an orogenic formation spanning from western Europe to eastern Asia. From west to east, a 
490 was identified in continental Europe above the Alps, spanning from western Europe to the

491 Balkan Peninsula.

492

493 Isolation by distance in Europe

494 In continental Europe, the shortest path overland did not correlate significantly with genetic

495 distance (fig. S13) (mantel test, $p$-value $=0.193, R=0.20 /$ linear model, $p$-value $=0.26, R^{2}=$

496 0.012). On the contrary, when the geographic distance between populations included the

497 barrier formed by the Alps (i.e. the Italian population was connected to other populations via

498 the Greek Peninsula, itself connected to western Europe via northern Europe), both tests

499 were significant (mantel test, $p$-value $=0.002, R=0.68 /$ linear model, $p$-value $=1.3 \times 10^{-5}, R^{2}=$

500 0.507). 
503 The history of natural populations is shaped by the combination of landscape barriers and

504 climatic variations that isolate and mix lineages through their combined actions. Consistently

505 with previous work ${ }^{20}$, we show that barn owls colonized the Western Palearctic in a ring-like

506 fashion around the Mediterranean Sea, with one arm around the Levant and the second

507 throughout Europe. However, using whole genome sequences we found this colonization

508 actually predates the last glaciation and pinpoint a narrow secondary contact zone between

509 the two lineages in Anatolia rather than in the Balkans. In addition, we provide evidence that

510 barn owls recolonized Europe after the LGM from two distinct glacial refugia - a western one

511 in Iberia and an eastern in Italy - rather than a single one as it was previously thought. As

512 temperatures started rising, western and northern Europe were colonized by owls from the

513 Iberian Peninsula while, in the meantime, the eastern refugium population of Italy had spread

514 to the Balkans (fig. 4). The western and eastern glacial populations finally met in eastern

515 Europe. This complex history of populations questions the taxonomy of the multiple Tyto alba

516 subspecies, highlights the key roles of mountain ranges and large water bodies as barriers to

517 gene flow for a widespread bird and illustrates the power of population genomics in

518 unraveling intricate patterns.

520 Colonization of the Western Palearctic and gene flow in Anatolia

521 Our results show that two distinct barn owl genetic lineages surround the Mediterranean

522 Basin: one in the Levant, and a second in Europe (fig. 1b, 1c, 1d, 1f), likely connected via northern Africa. Supported by the higher and specific diversity of the basal population of each

524 arm (namely, IS and PT; table 1), these observations are consistent with the ring colonization 
529 is more convoluted (see next section).

530 In previous studies, the ancestry of Greek and Aegean populations was unclear, with a

531 hypothesized mixed origin between the European and Levant lineages ${ }^{20}$. This uncertainty was microsatellites), as the genomic data reported here clearly show that Greek and Aegean owls are genetically much closer to European than to Levant ones (fig. 1b, 1c, 1d, 1f). This observation indicates that the European lineage reached further east than previously assumed, allowing us to pinpoint the secondary contact zone between the European and Levant lineages to Anatolia, instead of the Balkans as it had been proposed. In Anatolia, the

538 Taurus and Zagros mountain ranges form an imposing barrier that appears to have stopped

539 the expansion of the Levant lineage both during the ring colonization and nowadays.

540 Despite the barrier, and although we do not see a complete admixture of the two lineages,

541 there is evidence of some gene flow. Indeed, the first migration in Treemix (fig. 1f) pointed to

542 a secondary contact between $\mathrm{CY}$ and $\mathrm{AE}$, consistent with the signals of admixture between

543 these populations (fig. 1a, 1b, 2). The admixture pattern however is restricted geographically

544 to this narrow region and does not permeate further into either of the lineages, as 545 surrounding populations (IS in the Levant and GR and SB in Europe) do not show signals of 546 admixture (fig. 1, 2). Thus, the migration between populations on both sides seems limited and possibly only occurs along a narrow corridor along the Turkish coast where only a few 
barn owls have been recorded ${ }^{62}$. Further analyses with samples from Anatolia should allow

to characterize in high resolution how and when admixture occurred in this region.

550

\section{1}

\section{Glacial refugia and recolonization of Europe}

552 Previous studies showed that barn owls survived the last glaciation by taking refuge in the

Iberian Peninsula and maybe even in emerged land in the Bay of Biscay ${ }^{21,42}$. The observed

distribution of diversity in Europe, and especially the specific makeup of the Italian

and a second in Italy, derived from the Iberian population before the glaciation (fig. 3a and

suitable for the species at the time, but also show that, due to the low levels of the Adriatic

Sea, the suitable surface extended to the west coast of the Balkans (LGM - fig. 3c). Crucially,

three of the barn owl's key prey also had glacial refugia in the Italian and Balkan peninsulas,

567 European populations (GR), or from northern Africa to Italy via Sicily. The latter would have glaciation (fig. 3c) 66,67,68.

570 With the warming following the LGM, Europe became gradually more suitable and, by the

571 mid-Holocene (6000 years ago), most of western and northern Europe were appropriate for 
barn owls (fig. 2b) as well as the common vole (Microtus arvalis) ${ }^{69}$. The genetic similarity between Iberian (PT) and northwestern populations (CH, FR, DK; fig. 1a, 1e) indicates that barn owls colonized these newly available regions from the Iberian refugium as previously thought ${ }^{42}$. The contribution from the Italian refugium to northern populations appears to have been hindered by the Alps (see next sections), as suggested by the higher genetic distance between them (fig. 1a, 1c, 1d and 2). Instead, at this time the Adriatic Sea had neared today's levels, isolating genetically and geographically the Italian refugium from its component in the Balkan Peninsula (fig. 3b - IT-GR split 6k fig. 3b and fig. 3c). Only more recently did the rise of temperatures allow for areas in the east of Europe to become suitable, populations in the northeastern part of Europe (fig. 3c). In particular, the high heterozygosity and admixed ancestry of Serbian individuals (table 1, fig. 1a) suggest that the suture between the Iberian and the Italo-Greek glacial lineages took place in eastern Europe. This newly identified postglacial recolonization scheme of continental Europe by the barn owl matches the general pattern described for the brown bear (Ursus arctos) and shrew (Sorex sp.) ${ }^{7}$. zone (dark blue in GR and light blue in $\mathrm{CH}$ and DK, fig. 1a). Finally, the measure of haplotype sharing decreases consistently with distance between populations around the northern side of the Alps between populations from Iberia to Greece, excluding IT (fig. 2). Surrounded by the sea and the Alps, Italy is the exception and appears to have avoided incoming gene flow 
(own cluster in sNMF K>4, fig. S5). In contrast, the Balkan component admixes smoothly with

the other European populations. Such seamless mixing of the two glacial lineages from

600

602 Traditionally, in Europe, the eastern barn owl (T. a. guttata (Brehm, CL, 1831)) is defined by

its dark rufous ventral plumage in contrast to the white western barn owl (T. a. alba (Scopoli, 1769)) ${ }^{71}$. With a wide distribution, it is recorded from The Netherlands to Greece, including

615 The partition of genetic diversity among barn owls in the Western Palearctic allowed us to identify barriers and corridors to gene flow. Populations isolated by large water bodies have

617 accumulated substantial genetic differences as, for example, the higher $F_{\mathrm{ST}}$ in the Canary and 618 Cyprus islands (fig. 1c, table 1), and reflect the importance of water as a barrier to dispersion 619 in this species ${ }^{21}$. On the mainland, and as described for the American barn owl ${ }^{73}$, major 
mountain ranges act as significant obstacles to migration for European barn owls and can

621 generate genetic structure. First, the high mountain ranges of Taurus and Zagros coincide with the contact zone between the Levant and the European lineages both nowadays and potentially at the time of the pre-glacial ring colonization of Europe (see above). Second, the Alps and the Balkan Mountains slowed the northward expansion of the glacial populations of sides of their ranges. If these results remain to be confirmed with observational data (i.e. ringing data not available for all countries), they emphasize that, despite its worldwide repartition and its presence on many islands, the connectivity of barn owl populations is heavily driven by biogeographical barriers.

Conclusion

The combination of whole genome sequencing and sophisticated modeling methods revealed unachievable. It allowed the localization of a secondary contact zone as well as the discovery relevant samples to be collected and analyzed: What role did northern African populations played in connecting the Levant and European lineages? Did they contribute to the diversity observed in Italy? How narrow is the contact zone between the Levant and European lineages in Anatolia? Lastly, the origin of barn owls from the Western Palearctic as a whole also

641 deserves further investigation, as they are believed to have colonized the Western Palearctic 
compared to the Levant one. Such inconsistency points to the need for samples from around

645 the world, to understand how this charismatic group of nocturnal predators conquered the

646 entire planet.

647 Research on postglacial recolonization and the subsequent phylogeographic patterns peaked

648 at the turn of the century, with many studies providing an overview of the history of a wide

649 variety of organisms (reviewed by Hewitt ${ }^{7}$ ). The rise in availability of genomic data for non-

650 model species, combined with the type of approaches used here, will rewrite the history of

651 many of them. Furthermore, it will allow to detail the genomic consequences of such history

652 both from a neutral and selective perspective. Applied to several species, these approaches

653 will redefine with greater clarity the broad phylogeographical patterns in the Western

654 Palearctic and elsewhere, to re-think taxonomic classifications and to better understand how

655 organisms might adapt to a changing environment in a complex, fragmented and rapidly

656 changing landscape. 
659 We thank the following institutions and individuals for providing samples or aiding in 660 sampling: The European Barn Owl Network, Sylvain Antoniazza and Reto Burri, the Natural

661 History Museum of Tenerife, the Wildlife Rehabilitation Centre La Tahonilla (Cabildo Insular

662 de Tenerife), Kristijan Ovari and the Palić Zoo (Serbia). We thank Céline Simon and Luis San-

663 José for their valuable assistance with molecular work as well as Alexandros Topaloudis for scripts. Finally, we also thank Olivier Delaneau for his advice on the phasing of the individuals and its evaluation. This study was funded by the Swiss National Science Foundation with grants 31003A-138180 \& 31003A_179358 to JG and 31003A_173178 to AR.

$\underline{\text { Data Accessibility }}$

669 The raw Illumina reads for the whole-genome sequenced individuals are available in

670 BioProject PRJNA700797 and BioProject PRJNA727977.

671

672 Author Contribution

673

674 TC, APM, AR, JG designed this study; GD and APM produced whole-genome resequencing 675 libraries and called the variants; TC and APM conducted the analyses; KD, RL, JL, HDM, PB, 676 VB, MC, KD, HDM, NK, RL, FM, KO, LP, MR and FS provided samples to the study; TC led the 677 writing of the manuscript with input from APM and all authors. 
1. Huntley, B. \& Webb, T. Migration: Species' Response to Climatic Variations Caused by Changes in the Earth's Orbit. J. Biogeogr. 16, 5-19 (1989).

2. Irwin, D. E., Irwin, J. H. \& Price, T. D. Ring species as bridges between microevolution and speciation. Genetica 112, 223-243 (2001).

3. Devitt, T. J., Baird, S. J. \& Moritz, C. Asymmetric reproductive isolation between terminal forms of the salamander ring species Ensatina eschscholtzii revealed by fine-scale genetic analysis of a hybrid zone. BMC Evol. Biol. 11, 245 (2011).

686

4. Pereira, R. J., Monahan, W. B. \& Wake, D. B. Predictors for reproductive isolation in a ring species complex following genetic and ecological divergence. BMC Evol. Biol. 11, 194 (2011).

690

5. Cacho, N. I. \& Baum, D. A. The Caribbean slipper spurge Euphorbia tithymaloides: the first example of a ring species in plants. Proc. R. Soc. B Biol. Sci. 279, 3377-3383 (2012).

691

6. Stöck, M. et al. Cryptic diversity among Western Palearctic tree frogs: Postglacial range expansion, range limits, and secondary contacts of three European tree frog lineages (Hyla arborea group). Mol. Phylogenet. Evol. 65, 1-9 (2012).

7. Hewitt, G. M. Post-glacial re-colonization of European biota. Biol. J. Linn. Soc. 68, 87-112 (1999).

696

8. Hewitt, G. The genetic legacy of the Quaternary ice ages. Nature 405, 907-913 (2000).

697

9. Willeit, M., Ganopolski, A., Calov, R. \& Brovkin, V. Mid-Pleistocene transition in glacial cycles explained by declining CO2 and regolith removal. Sci. Adv. 5, eaav7337 (2019).

10. Clark, P. U. et al. The Last Glacial Maximum. Science 325, 710-714 (2009).

700

11. Can secondary contact following range expansion be distinguished from barriers to gene flow? [PeerJ]. https://peerj.com/articles/5325/.

702

12. Boucher, F. C., Zimmermann, N. E. \& Conti, E. Allopatric speciation with little niche divergence is common among alpine Primulaceae. J. Biogeogr. 12 (2015).

704

13. Tomasello, S., Karbstein, K., Hodač, L., Paetzold, C. \& Hörandl, E. Phylogenomics unravels Quaternary vicariance and allopatric speciation patterns in temperate-montane plant species: A case study on the Ranunculus auricomus species complex. Mol. Ecol. 29, 20312049 (2020).

708

14. Capblancq, T., Després, L., Rioux, D. \& Mavárez, J. Hybridization promotes speciation in Coenonympha butterflies. Mol. Ecol. 24, 6209-6222 (2015).

15. Hey, J. The Divergence of Chimpanzee Species and Subspecies as Revealed in Multipopulation Isolation-with-Migration Analyses. Mol. Biol. Evol. 27, 921-933 (2010).

16. Linck, E., Freeman, B. G. \& Dumbacher, J. P. Speciation and gene flow across an elevational gradient in New Guinea kingfishers. J. Evol. Biol. 33, 1643-1652 (2020).

17. Poelstra, J. W. et al. The genomic landscape underlying phenotypic integrity in the face of gene flow in crows. Science 344, 1410-1414 (2014). mutation load in a broadly distributed Pacific Salmon. PLOS Genet. 16, e1008348 (2020). 
19. Duranton, M. et al. The origin and remolding of genomic islands of differentiation in the European sea bass. Nat. Commun. 9, 2518 (2018).

20. Burri, R. et al. The genetic basis of color-related local adaptation in a ring-like colonization around the Mediterranean: GENETICS OF CIRCUM-MEDITERRANEAN COLOR ADAPTATION. Evolution 70, 140-153 (2016).

21. Unexpected post-glacial colonisation route explains the white colour of barn owls (Tyto alba) from the British I bioRxiv. https://www.biorxiv.org/content/10.1101/2021.04.23.441058v1.

22. Van der Auwera, G. A. et al. From FastQ data to high confidence variant calls: the Genome Analysis Toolkit best practices pipeline. Curr. Protoc. Bioinforma. 43, 11.10.1-11.10.33 (2013).

23. Trimmomatic: a flexible trimmer for Illumina sequence data | Bioinformatics | Oxford Academic. https://academic.oup.com/bioinformatics/article/30/15/2114/2390096.

24. Li, H. \& Durbin, R. Fast and accurate short read alignment with Burrows-Wheeler transform. Bioinforma. Oxf. Engl. 25, 1754-1760 (2009).

25. ANGSD: Analysis of Next Generation Sequencing Data | BMC Bioinformatics | Full Text. https://bmcbioinformatics.biomedcentral.com/articles/10.1186/s12859-014-0356-4.

26. Danecek, P. et al. The variant call format and VCFtools. Bioinforma. Oxf. Engl. 27, 21562158 (2011).

27. Graffelman, J. \& Camarena, J. M. Graphical tests for Hardy-Weinberg equilibrium based on the ternary plot. Hum. Hered. 65, 77-84 (2008).

28. Graffelman, J. Exploring Diallelic Genetic Markers: The HardyWeinberg Package. J. Stat. Softw. 64, 1-23 (2015).

29. Team, R. C. R: A language and environment for statistical computing (3.5. 1)[Computer software]. R Foundation for Statistical Computing. (2020).

30. Martin, M. et al. WhatsHap: fast and accurate read-based phasing. bioRxiv 085050 (2016) doi:10.1101/085050.

31. Delaneau, O., Zagury, J.-F., Robinson, M. R., Marchini, J. L. \& Dermitzakis, E. T. Accurate, scalable and integrative haplotype estimation. Nat. Commun. 10, 5436 (2019).

32. Choi, Y., Chan, A. P., Kirkness, E., Telenti, A. \& Schork, N. J. Comparison of phasing strategies for whole human genomes. PLOS Genet. 14, e1007308 (2018).

33. Fast and Efficient Estimation of Individual Ancestry Coefficients. https://www.ncbi.nlm.nih.gov/pmc/articles/PMC3982712/.

34. PLINK: A Tool Set for Whole-Genome Association and Population-Based Linkage Analyses. https://www.ncbi.nlm.nih.gov/pmc/articles/PMC1950838/.

35. Pickrell, J. K. \& Pritchard, J. K. Inference of Population Splits and Mixtures from GenomeWide Allele Frequency Data. PLoS Genet. 8, e1002967 (2012).

36. Weir, B. S. \& Goudet, J. A Unified Characterization of Population Structure and Relatedness. Genetics 206, 2085-2103 (2017).

37. Zheng, X. et al. A high-performance computing toolset for relatedness and principal component analysis of SNP data. Bioinformatics 28, 3326-3328 (2012). 
38. Goudet, J. hierfstat, a package for $r$ to compute and test hierarchical F-statistics. Mol. Ecol. Notes 5, 184-186 (2005).

39. Lawson, D. J., Hellenthal, G., Myers, S. \& Falush, D. Inference of Population Structure using Dense Haplotype Data. PLoS Genet. 8, e1002453 (2012).

40. Excoffier, L. \& Foll, M. fastsimcoal: a continuous-time coalescent simulator of genomic diversity under arbitrarily complex evolutionary scenarios. Bioinformatics 27, 1332-1334 (2011).

41. Excoffier, L., Dupanloup, I., Huerta-Sánchez, E., Sousa, V. C. \& Foll, M. Robust Demographic Inference from Genomic and SNP Data. PLOS Genet. 9, e1003905 (2013).

42. Antoniazza, S. et al. Natural selection in a postglacial range expansion: the case of the colour cline in the European barn owl. Mol. Ecol. 23, 5508-5523 (2014).

43. Background selection and biased gene conversion affect more than $95 \%$ of the human genome and bias demographic inferences | elife. https://elifesciences.org/articles/36317.

44. Uva, V., Päckert, M., Cibois, A., Fumagalli, L. \& Roulin, A. Comprehensive molecular phylogeny of barn owls and relatives (Family: Tytonidae), and their six major Pleistocene radiations. Mol. Phylogenet. Evol. 125, 127-137 (2018).

45. Akaike, H. A New Look at the Statistical Model Identification. in Selected Papers of Hirotugu Akaike (eds. Parzen, E., Tanabe, K. \& Kitagawa, G.) 215-222 (Springer, 1998). doi:10.1007/978-1-4612-1694-0_16.

46. Kocher, T. D. et al. Dynamics of mitochondrial DNA evolution in animals: amplification and sequencing with conserved primers. Proc. Natl. Acad. Sci. 86, 6196-6200 (1989).

47. Malaspinas, A.-S. et al. A genomic history of Aboriginal Australia. Nature 538, 207-214 (2016).

48. Elith, J. et al. A statistical explanation of MaxEnt for ecologists. Divers. Distrib. 17, 43-57 (2011).

49. M. Exposito-Alonso. rbioclim: Improved getData function from the raster R package to interact with past, present and future climate data from worldclim.org. (2017).

50. Warren, D. L. \& Seifert, S. N. Ecological niche modeling in Maxent: the importance of model complexity and the performance of model selection criteria. Ecol. Appl. 21, 335342 (2011).

51. BirdLife International. The IUCN Red List of Threatened Species. Version 6.2. (2019).

52. Fourcade, Y. Comparing species distributions modelled from occurrence data and from expert-based range maps. Implication for predicting range shifts with climate change. Ecol. Inform. 36, 8-14 (2016).

53. Swets, J. A. Measuring the accuracy of diagnostic systems. Science 240, 1285-1293 (1988).

54. Li, Y., Li, M., Li, C. \& Liu, Z. Optimized Maxent Model Predictions of Climate Change Impacts on the Suitable Distribution of Cunninghamia lanceolata in China. Forests 11, 302 (2020).

55. Petkova, D., Novembre, J. \& Stephens, M. Visualizing spatial population structure with estimated effective migration surfaces. Nat. Genet. 48, 94-100 (2016). 
800

801

802

803

804

805

806

807

808

809

810

811

812

813

814

815

816

817

818

819

820

821

822

823

824

825

826

827

828

829

830

831

832

833

834

835

836

837

838

56. Dray, S. \& Dufour, A.-B. The ade4 Package: Implementing the Duality Diagram for Ecologists. J. Stat. Softw. 22, 1-20 (2007).

57. Lourenço, R., Roque, I., Tomé, R. \& Sepúlveda, P. Current status and distribution of nocturnal birds (Strigi- formes and Caprimulgiformes) in Portugal. 16.

58. Martí R and del Moral C (eds). Atlas de las aves reproductoras de España. (2003).

59. Ornitologia Italiana - Vol.3 - Stercorariidae Caprimulgidae - Brichetti Pierandrea; Fracasso Giancarlo | Libro Alberto Perdisa Editore 06/2005 - HOEPLI.it. www.hoepli.it https://www.hoepli.it/libro/ornitologia-italiana-vol3-stercorariidaecaprimulgidae/9788883722417.html.

60. 9783952106457: Atlas des oiseaux nicheurs de Suisse : Distribution des oiseaux nicheurs en Suisse et au Liechtenstein en 1993-1996 - AbeBooks - Hans Schmid; Roland Luder; Beat Naef-Daenzer; Roman Graf; Niklaus Zbinden: 3952106453. https://www.abebooks.fr/9783952106457/Atlas-oiseaux-nicheurs-Suisse-Distribution3952106453/plp.

61. Birds in Europe: Population Estimates, Trends and Conservation Status | BirdLife. https://www.birdlife.org/europe-and-central-asia/news/birds-europe-populationestimates-trends-and-conservation-status.

62. Göçer, E. \& Johnson, D. H. The Barn owl (Tyto alba) in Turkey. 7(3-4): 500-506, (2018).

63. Stojak, J., Borowik, T., Górny, M., McDevitt, A. D. \& Wójcik, J. M. Climatic influences on the genetic structure and distribution of the common vole and field vole in Europe. Mammal Res. 64, 19-29 (2019).

64. Herman, J. S. et al. Post-glacial colonization of Europe by the wood mouse, Apodemus sylvaticus: evidence of a northern refugium and dispersal with humans. Biol. J. Linn. Soc. $\mathrm{n} / \mathrm{a}$.

65. Dubey, S. et al. Molecular evidence of Pleistocene bidirectional faunal exchange between Europe and the Near East: the case of the bicoloured shrew (Crocidura leucodon, Soricidae). J. Evol. Biol. 20, 1799-1808 (2007).

66. Dapporto, L. \& Bruschini, C. Invading a refugium: post glacial replacement of the ancestral lineage of a Nymphalid butterfly in the West Mediterranean. Org. Divers. Evol. 12, 39-49 (2012).

67. Husemann, M., Schmitt, T., Zachos, F. E., Ulrich, W. \& Habel, J. C. Palaearctic biogeography revisited: evidence for the existence of a North African refugium for Western Palaearctic biota. J. Biogeogr. 41, 81-94 (2014).

68. Lodolo, E. et al. Post-LGM coastline evolution of the NW Sicilian Channel: Comparing highresolution geophysical data with Glacial Isostatic Adjustment modeling. PLOS ONE 15, e0228087 (2020).

69. Heckel, G., Burri, R., Fink, S., Desmet, J.-F. \& Excoffier, L. Genetic Structure and Colonization Processes in European Populations of the Common Vole, Microtus Arvalis. Evolution 59, 2231-2242 (2005). 
70. Antoniazza, S., Burri, R., Fumagalli, L., Goudet, J. \& Roulin, A. Local adaptation maintains Clinal variation in melanin-based coloration of Euopean barn owl (Tyto alba). Evolution (2010) doi:10.1111/j.1558-5646.2010.00969.x.

842 71. Mátics, R., Hoffmann, G. \& Owl, B. Location of the transition zone of the Barn Owl subspecies Tyto alba alba and Tyto alba guttata (Strigiformes: Tytonidae). (2001).

844 72. Clements, J. F. et al. The eBird/Clements Checklist of Birds of the World: v2019. (2019).

845 73. Machado, A. P., Clément, L., Uva, V., Goudet, J. \& Roulin, A. The Rocky Mountains as a dispersal barrier between barn owl (Tyto alba) populations in North America. J. Biogeogr. 45, 1288-1300 (2018). 
Table 1 - Population genetic diversity, inbreeding and divergence estimates for 11 populations of barn owls from the Western Palearctic. Standard deviations of the mean are provided between brackets for each parameter, see Methods section for details.

855

\begin{tabular}{|c|c|c|c|c|c|c|c|}
\hline Pop & $N$ & $\# P I$ & $\# P A$ & \#Rare & $F_{I T}$ & $F_{I S}$ & Pop $F_{S T}$ \\
\hline$W C$ & 9 & $\begin{array}{c}2217235 \\
(37007)\end{array}$ & $\begin{array}{c}123016 \\
(2465)\end{array}$ & $\begin{array}{l}407403 \\
(10568)\end{array}$ & $0.067(0.054)$ & $-0.022(0.057)$ & $0.116(0.006)$ \\
\hline$P T$ & 9 & $\begin{array}{c}2639343 \\
(22857)\end{array}$ & $\begin{array}{c}102252 \\
(2305)\end{array}$ & $\begin{array}{l}539157 \\
(10106)\end{array}$ & $-0.018(0.042)$ & $-0.008(0.042)$ & $0.003(0.003)$ \\
\hline$F R$ & 4 & $\begin{array}{c}2151627 \\
(0)\end{array}$ & $\begin{array}{l}30290 \\
(581)\end{array}$ & $\begin{array}{c}287746 \\
(1468)\end{array}$ & $0.039(0.124)$ & $0.043(0.131)$ & $0.050(0.005)$ \\
\hline $\mathrm{CH}$ & 10 & $\begin{array}{c}2494462 \\
(10723)\end{array}$ & $\begin{array}{c}47532 \\
(730)\end{array}$ & $\begin{array}{c}386654 \\
(3117)\end{array}$ & $0.025(0.019)$ & $-0.011(0.019)$ & $0.036(0.002)$ \\
\hline$D K$ & 10 & $\begin{array}{c}2410615 \\
(15558)\end{array}$ & $\begin{array}{l}40650 \\
(1378)\end{array}$ & $\begin{array}{c}349800 \\
(5434)\end{array}$ & $0.026(0.020)$ & $-0.02(0.021)$ & $0.049(0.002)$ \\
\hline$I T$ & 9 & $\begin{array}{c}2404069 \\
(7267)\end{array}$ & $\begin{array}{c}66297 \\
(1638)\end{array}$ & $\begin{array}{c}401842 \\
(4483)\end{array}$ & $0.035(0.012)$ & $-0.022(0.012)$ & $0.052(0.005)$ \\
\hline$S B$ & 5 & $\begin{array}{c}2336060 \\
(0)\end{array}$ & $\begin{array}{l}32096 \\
(1515)\end{array}$ & $\begin{array}{c}326906 \\
(2519)\end{array}$ & $0.025(0.011)$ & $-0.038(0.011)$ & $0.056(0.004)$ \\
\hline$G R$ & 9 & $\begin{array}{c}2454653 \\
(7365)\end{array}$ & $\begin{array}{l}44996 \\
(1146)\end{array}$ & $\begin{array}{c}378422 \\
(4152)\end{array}$ & $0.018(0.028)$ & $-0.016(0.027)$ & $0.039(0.002)$ \\
\hline$A E$ & 10 & $\begin{array}{c}2460422 \\
(20650)\end{array}$ & $\begin{array}{l}56260 \\
(3439)\end{array}$ & $\begin{array}{l}403259 \\
(10465)\end{array}$ & $0.018(0.060)$ & $-0.001(0.062)$ & $0.030(0.002)$ \\
\hline$C Y$ & 10 & $\begin{array}{c}2338318 \\
(48463)\end{array}$ & $\begin{array}{l}113377 \\
(2229)\end{array}$ & $\begin{array}{l}480021 \\
(13581)\end{array}$ & $0.022(0.047)$ & $-0.034(0.049)$ & $0.059(0.004)$ \\
\hline IS & 9 & $\begin{array}{c}2509099 \\
(9500)\end{array}$ & $\begin{array}{c}172624 \\
(4018)\end{array}$ & $\begin{array}{c}608944 \\
(3597)\end{array}$ & $-0.019(0.015)$ & $-0.036(0.016)$ & $0.021(0.003)$ \\
\hline
\end{tabular}

$\mathrm{N}$ : number of individuals in the population; \#PI: number of polymorphic sites per populations; \#PA: number of private alleles per population; \#Rare: Number of rare alleles per population; $F_{\mathrm{IT}}$ : mean individual inbreeding coefficient relative to the meta-population; $F_{\text {IS: }}$ population level inbreeding coefficient; $F_{\text {ST: }}$ population specific $F_{\text {ST }}$ as in Weir and Goudet 2017. Populations: WC - Canary Islands, PT - Portugal, FR - France, $\mathrm{CH}$ - Switzerland, DK Denmark, IT - Italy, SB - Serbia, GR - Greece, AE - Aegean Islands, CY - Cyprus, IS - Israel. 
a

b

\section{a}

\section{(2)}

\section{.}
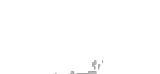

s.

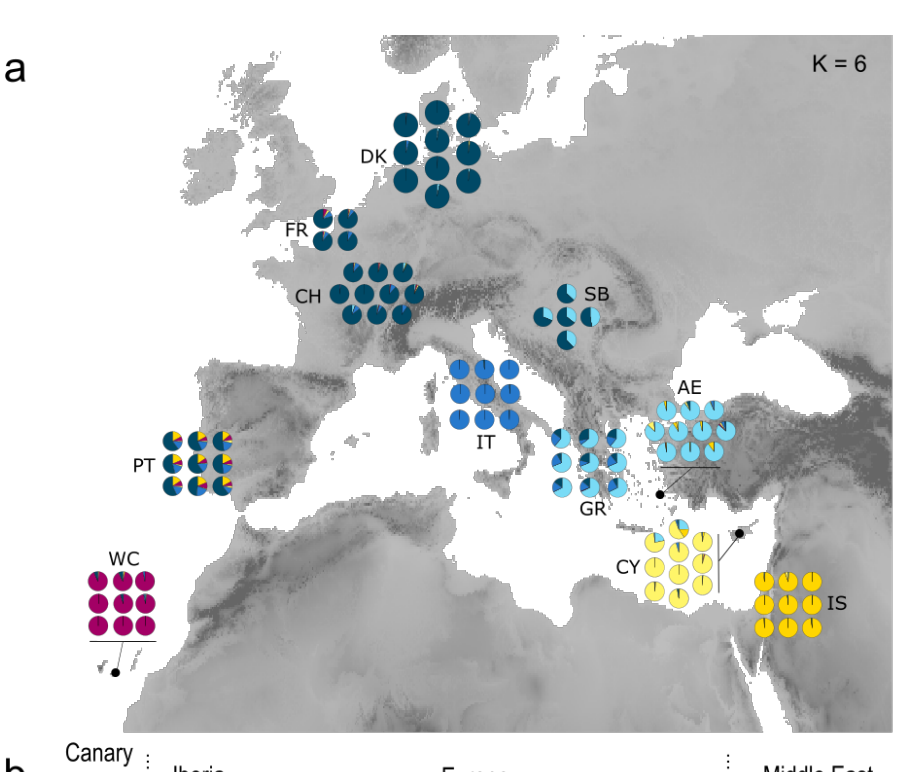

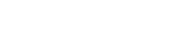

II
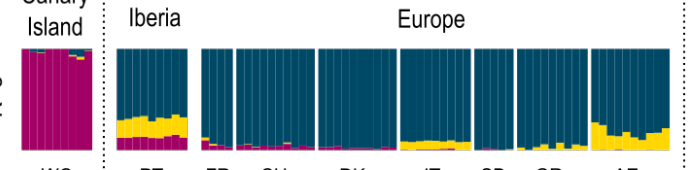

Middle East
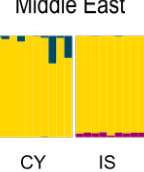

\section{C}

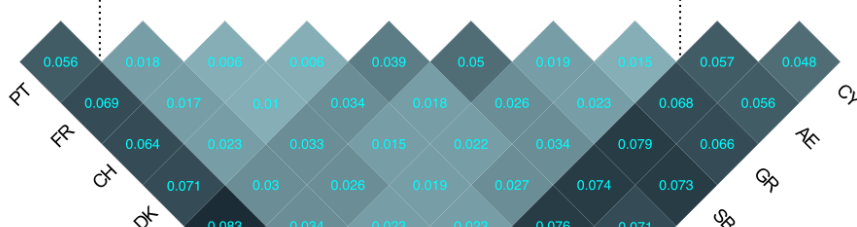

e
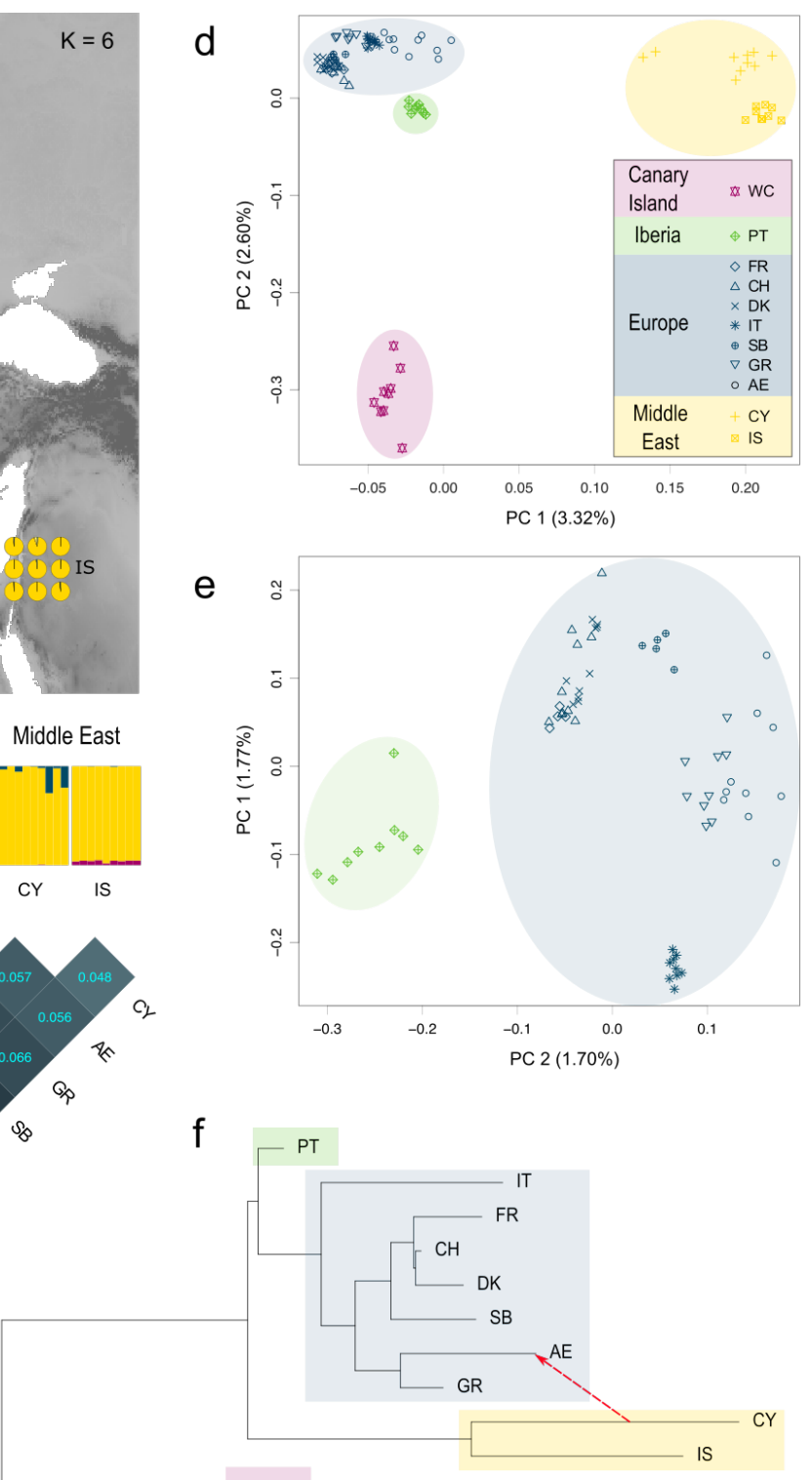

Figure 1 - Genetic structure of barn owl populations in Western Palearctic. (a) Population structure for $\mathrm{K}=6$. Pie charts denote the individual proportion of each of lineages as determined by sNMF and are located at the approximate centroid of the sampled population. (b) Population structure for $\mathrm{K}=3$. Each bar denotes the individual proportion of each of the 3 lineages as determined by sNMF. (c) Matrix of pairwise $F_{\text {ST }}$ between barn owl populations in Western Palearctic. The heatmap provides a visual representation of the $F_{\text {ST }}$ values given in each cell. (d) PCA based on full set of 94 individuals. Point shape denotes populations and colored circles enclose sample clusters observed in SNMF $(K=3)$. Values in parentheses indicate the percentage of variance explained by each axis. (e) PCA based on of the 66 European individuals. (f) Population tree and the first migration event in Western Palearctic populations inferred by Treemix. 


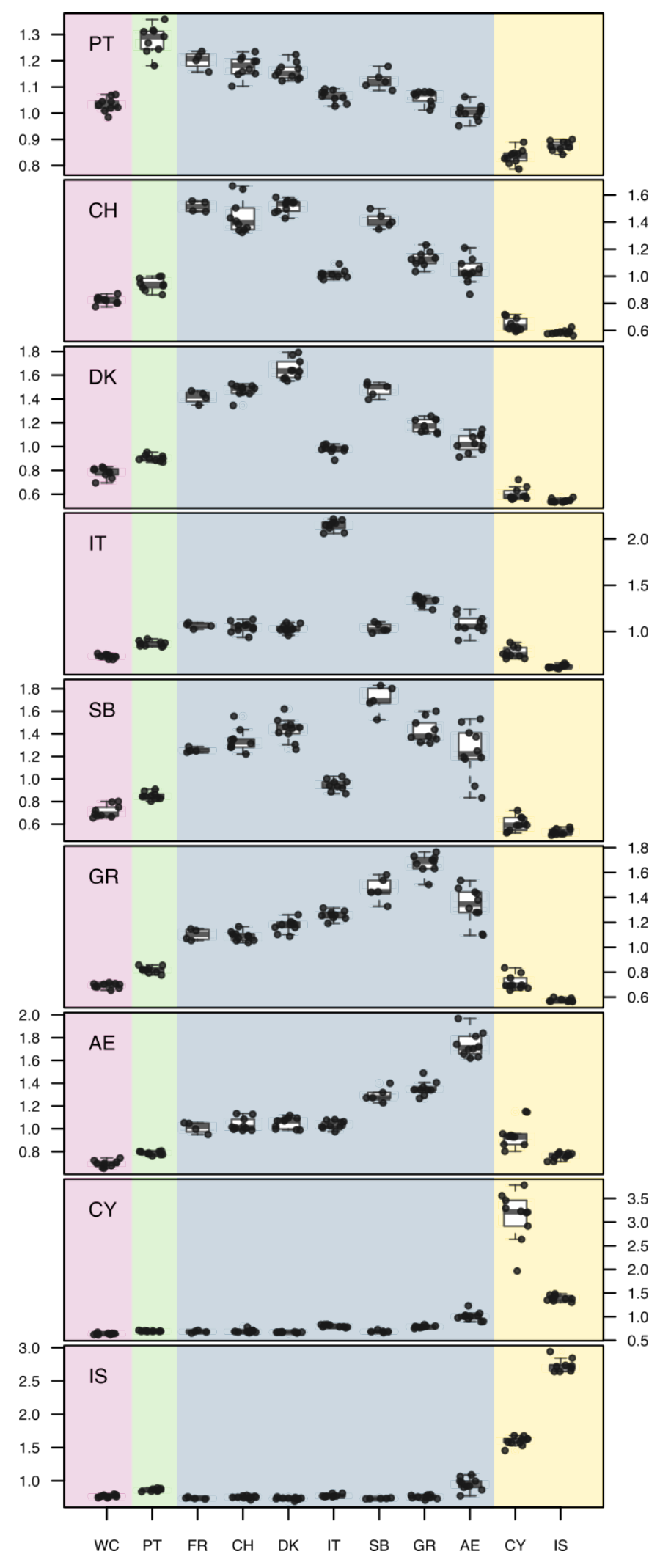

Figure 2 - Individual haplotype sharing between barn owl populations. Part of the total length of ChromoPainter chunks inherited from other genomes. Each graph summarizes the information of all the genomes from a given population, indicated on the top-left corner. Background colors match the lineages identified in fig. 1. 


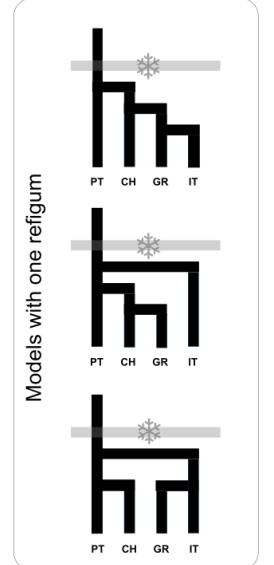

b

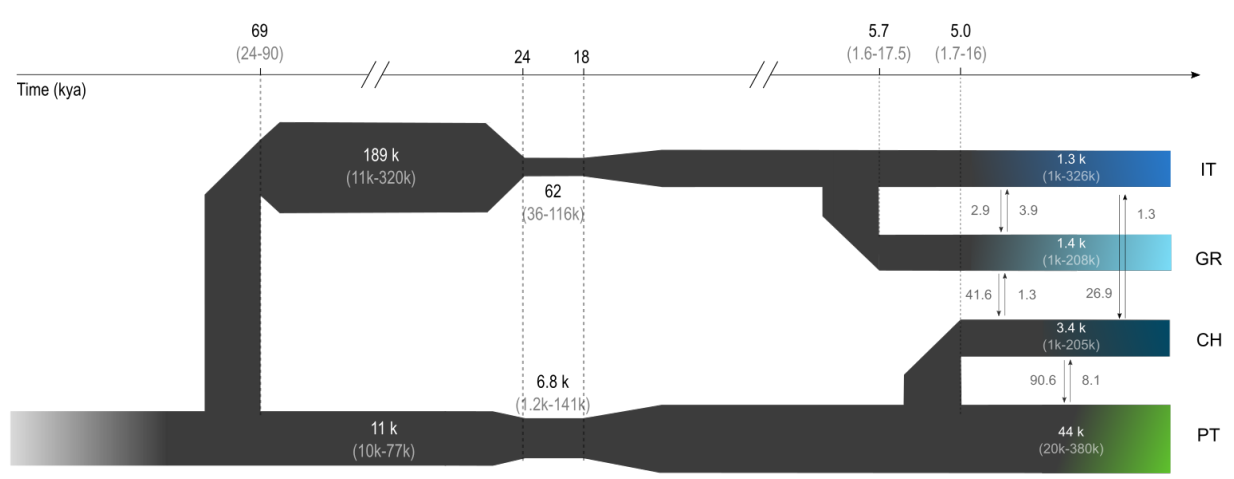

C

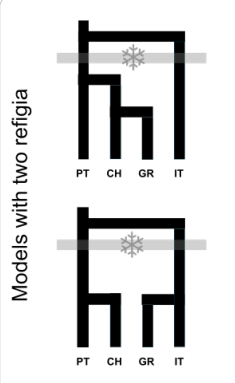

887

888

889

890

891

892

893

894

895

896

897

898

899

900

901

902

903

904

905

906

Figure 3 - Modeling of the history of the barn owl in Europe. (a) Schematic representation of the five demographic scenarios tested for the colonization of the Europe by barn owls. Three models included one refugium in the Iberia during the LGM while the last two included two refugia, one in Iberia and the second in Italy. Grey bars with snowflakes represent the last glaciation. (b) Best supported demographic model for the history of European barn owl populations as determined by fastsimcoal2. Time is indicated in thousands of years, determined using a 3-year generation time, confidence intervals at $95 \%$ are given between brackets. Population sizes (haploid) are shown inside each population bar; arrows indicate forward-in-time migration rate and direction. (c) Species distribution model of barn owls based on climatic variables, projected into the past (last glacial maximum (20 kya) mid-Holocene (6 kya)) and today's condition. Locations in dark grey were highly suitable in $90 \%$ of the models. Below that threshold cells were considered as unsuitable (lightest grey shade on the graph). The present coastline is outlined in blue in all graphs. 


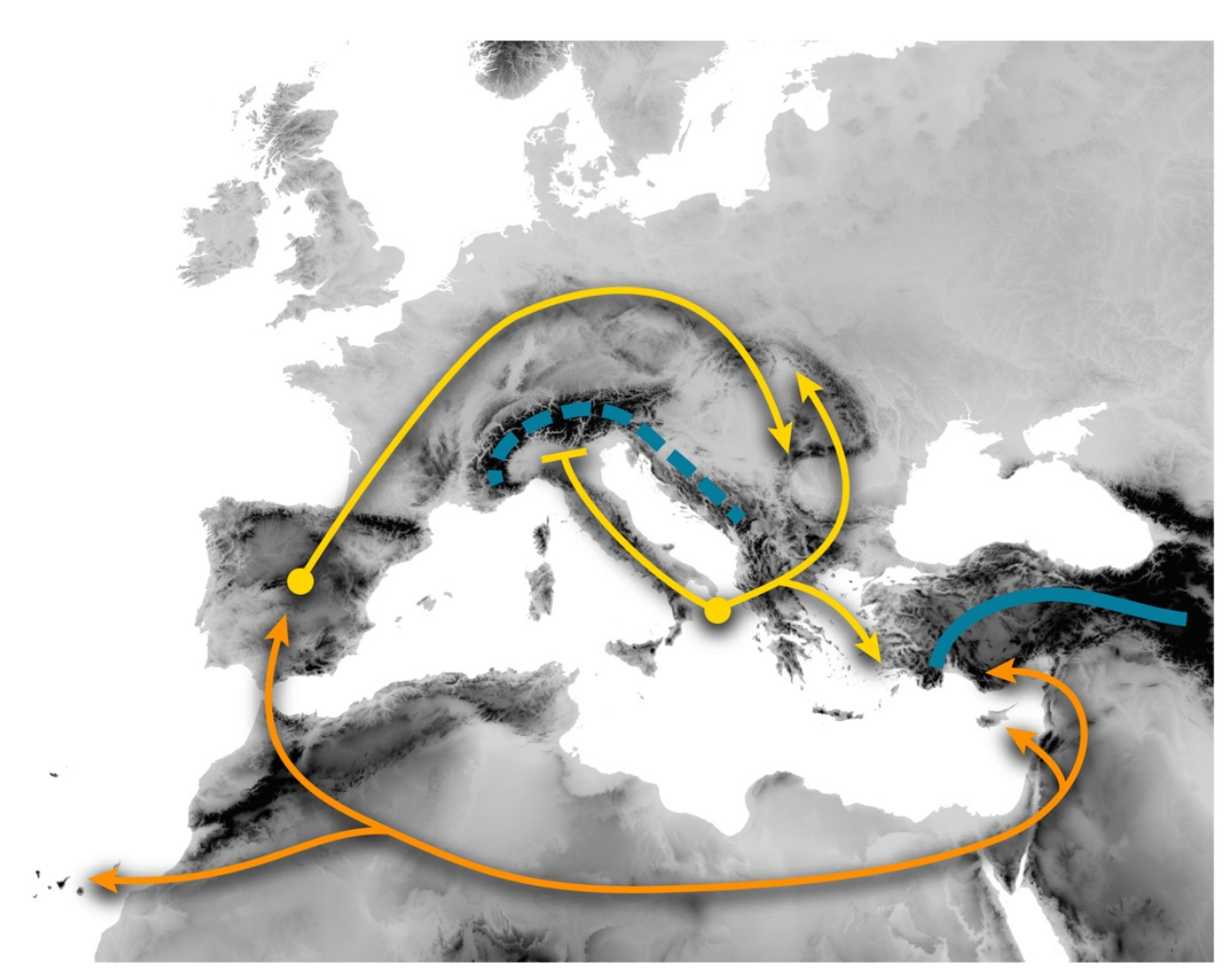

Figure 4 - Schematic representation of the history of barn owls in the Western Palearctic 911 and the main barriers in the region. Orange arrows depict the colonization of the region by the three main lineages (Levant, Canary Islands, European). Yellow arrows represent the modeled postglacial recolonization scheme of Europe, with two distinct refugia (yellow dots). Blue lines represent the main barriers identified in this work, namely the Alps in

915 Europe (dashed line) and the Taurus and Zagros mountains in Anatolia (solid line). 